Journal of ISMAC (2019)

Vol.01/ No. 01

Pages: $160-171$

http://irojournals.com/iroismac/

DOI: https://doi.org/10.36548/jismac.2019.3.003

\title{
ENHANCED NETWORK SELECTION AND HANDOVER SCHEMA FOR HETEROGENEOUS WIRELESS NETWORKS
}

\author{
Dr. M. Durai Pandian, \\ Professor, Department of CSE, \\ Vivekanandha College of Technology for Women, India. \\ Email id: svsduraipandian@gmail.com
}

\begin{abstract}
The seamless communication between the devices of the heterogeneous wireless networks still remains as a challenge due to the delay incurred, the cost difference and the bandwidth variations.so it becomes necessary for a perfect management of the hand over and the selection of the network to provide a continuous conveyance in the broadcasting or the sharing of the information's. So the paper initiates an enhanced network selection and a vertical hand over schema that is context aware and based on the user preference utilizing the grey relational analysis integrated with the particle swarm optimization. The performance analysis of the proposed method of vertical handover evinces the perfect network selection that enables a continuous connection for the heterogeneous networks and shows an enhanced throughput and latency in handover compared to the other methods like TOPSIS (technique for order preference by similarity to ideal solution) and SAW(simple additive weighting).
\end{abstract}

Keywords: Vertical handover, Heterogeneous wireless networks, Grey rational analysis, Particle swarm optimization, Network selection

\section{INTRODUCTION}

The wireless networks are unlike wired network that provide cables for connecting of devices and establishing communication between them, the wireless network uses the radio waves to provide communication between the devices and enable them to be connected using the wireless data connections. The wireless networks enable cost effective communication to a longer distance eluding the confusions that could be created when forming connections between the devices using the cables. The continuous reforms and the progress in the development of the metal oxide silicon semiconductor transistor led to the emerging of the digital wireless networks and the further reforms in the wireless networks made possible the developments of the base stations modules, telecommunications circuits etc. and the networks such as the $2 \mathrm{G}, 3 \mathrm{G}$ and the $4 \mathrm{G}$ and the upcoming $5 \mathrm{G}$ networks. Some of the well-known wireless networks that we come across in our daily lives are cellular network, wireless local area network, wireless 
Journal of ISMAC (2019)

Vol.01/ No. 01

Pages: $160-171$

http://irojournals.com/iroismac/

DOI: https://doi.org/10.36548/jismac.2019.3.003

sensor networks, satellite communication and microwave network that are terrestrial. The fig. 1 below shows the different types of the networks and the common types of networks available.

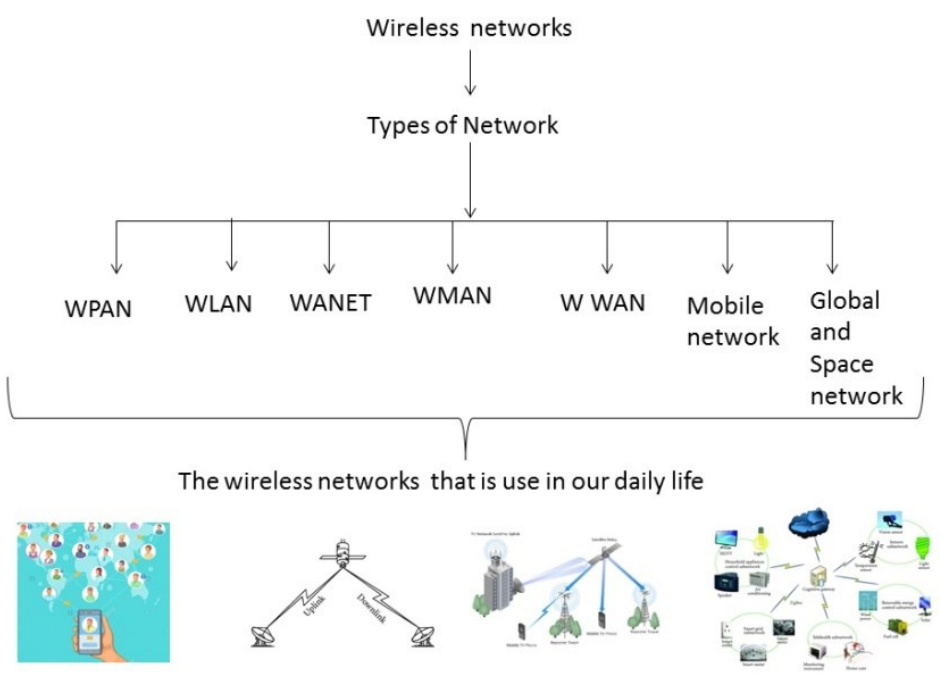

Fig .1 Wireless Networks

Though seeming to be efficient the wireless network endure certain difficulties due to the interference, absorption and reflection, multipath fading and the hidden node problem shared resource problem etc. so this necessitates for the perfect network selection that would provide with an optimal way of data transfer. Usually they arise as a very big problem that is difficult to solve in the cases of the wireless networks that are heterogeneous as the connections are established with devices that are enabled with a different operating systems and the protocols.

The next issue that arises in the wireless network is the handing- over complexities. The heterogeneous wireless networks does not face much problems in the horizontal hand offs as such that are faced in the homogenous networks. But the heterogeneous networks face difficulties in the vertical handover as they can be proceeded 
Journal of ISMAC (2019)

Vol.01/ No. 01

Pages: $160-171$

http://irojournals.com/iroismac/

DOI: https://doi.org/10.36548/jismac.2019.3.003

according to the users wish and their comfort. The main issues that are to be considered in the vertical handover are the seamless connection and the automatic network switching [6]. The fig.2 states the metrics and the decision making strategies that are for the vertical hand over.
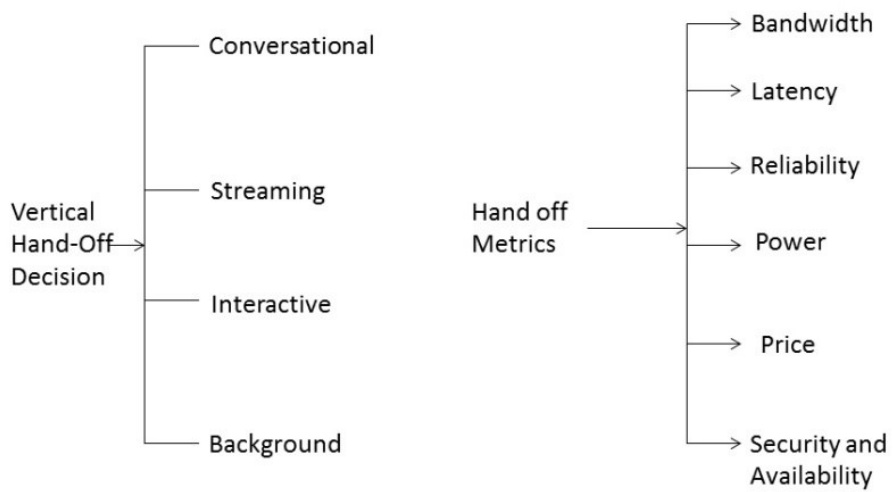

Fig.2. Hand over decision making and the Metrics involved

So he hand over becomes a necessary requirement for the wireless network that are heterogeneous, to enhance the quality of the service for the seamless connections [9].

So the paper proceeds with a proper enhanced vertical handover techniques followed by an optimal network selection to improve the communication providing a seamless connection and the automatic hand over.

The paper organization proceeds with the 2 detailing the related works, 3 providing the proposed work , 4 providing the performance analysis of the proposed work and 5 presenting the summary of the work done as a conclusion.

\section{RELATED WORKS}


Journal of ISMAC (2019)

Vol.01/ No. 01

Pages: $160-171$

http://irojournals.com/iroismac/

DOI: https://doi.org/10.36548/jismac.2019.3.003

Han et al [1] the author states that the failures in the wireless networks, in terms of the delay, power consumption and discontinuity in the information transfer could be overcome using the proper resource allocation for the wireless networks.

Fitzek et al [2] the book presents the details of the co-operation manner of the wireless communications, about the competitions, the cognitions, techniques, coding related to the wireless networks, along with the stability, security, power consumption and the spectrum usage of the wireless networks.

Khan et al [3] the author proposes the neighbor discovery for the conventional wireless networks and the cognitive radio networks presenting the basic taxonomy, challenges along with the future research directions.

Wang et al [4] the author details the mathematical models for selecting of the network that is optimal to reduce the congestion in it and provide with the best networks for the different types of communications at any time from any where

$\mathrm{Xu}$ et al [5] the author presents a comprehensive review for the integration of the wireless networks and the internet of things, along with the probable visions and the open challenges.

Kassar,et al [6] the author provides with the latest and the interesting methods of the hand over by classifying them into five categories, and presenting the essential characteristics of them along with the comparison of the various methodologies presented. The paper includes the handover management ideas in the heterogeneous network as shown in the fig 
Journal of ISMAC (2019)

Vol.01/ No. 01

Pages: $160-171$

http://irojournals.com/iroismac/

DOI: https://doi.org/10.36548/jismac.2019.3.003

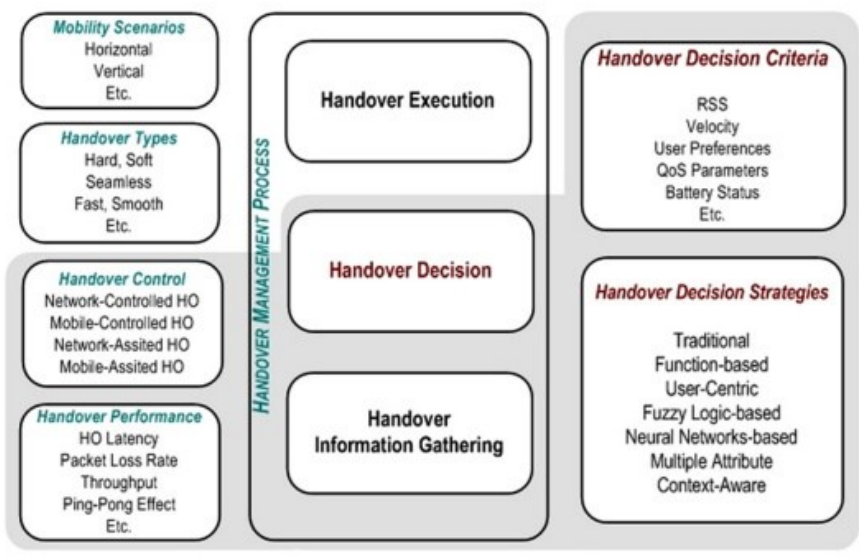

Fig Handover_Management Trends [6]

Stevens et al [7] the paper presents the comparison between the four various vertical handoffs such as the SAW, TOPSIS, MEW and GRA, and measures the characteristics of the all the algorithms based on the bandwidth, delay, packet loss rate, and cost. And from the results it was understood that the GRA provides with the lowest delay and the highest bandwidth.

Niyato et al [8] the author utilizes the theory of the evolutionary games for perfect network selection, to provide with a seamless connection and the high-speed wireless connectivity to provide with the perfect load balancing to elude the congestion in the network and the degradation in the performance. The author provides the study of the dynamics of the network selection in a heterogeneous network.

Yan et al [9] the paper provides with the survey that is comprehensive for the algorithms that are utilized in the vertical decision. The author in order to present the survey classifying the types of the hand over based on main handover decision that is used and evaluates the compromises among the complexity and the efficiency.

Stevens-Navarro et al [10] the paper utilizes the Markov decision algorithm for enabling a perfect handover to have a seamless connection in the information sharing. To provide a better performance in terms of the delay, bandwidth jitter, packet delivery rate etc. 
Journal of ISMAC (2019)

Vol.01/ No. 01

Pages: $160-171$

http://irojournals.com/iroismac/

DOI: https://doi.org/10.36548/jismac.2019.3.003

Zahran, et al [11] the author presents the network life span based hand-off and examines the consequence of the application based signal strength threshold in signaling load, bandwidth available and the packet delay.

Bari et al [12] the decision making process to rank the user networks based on the unique decision process for having best network access in transmitting of the information's was proffered in the paper.

Ormond et al [13] to have a multiple network access at the same time. The author proposes the user-centric solution to select wireless network that supports the data conveyance with the optimal performance. Providing a timely and a quality data delivery at a reduced cost.

Sgora, et al [14] for the selection of the optimal networks, the paper proffers the EANSA for the heterogeneous networks combining two multi-attribute decisions making to have a better network accessing.

Lei, et al [15] the author presents the review of the challenges incurred in the heterogeneous wireless networks when engaged with the mobile cloud computing due to the limitations of the bandwidth and the latencies in the communication.

Kennedy et al [16] the author describes the steps of the particle swarm optimization for achieving optimal results in the nonlinear functions along with its relationship with the genetic algorithm and the artificial life.

\section{PROPOSED WORK}

The heterogeneous network that is a type of wireless network supported by variety of access technologies for instance consider a communication between two devices with one under the wireless local area network and the other under the cellular network. The communication extended by switching from WLAN to cellular network is called a wireless heterogeneous network. This communication is often affected by the network channels aided with the limitations in the bandwidth and the latency incurred during communicating [15]. Especially on the instance when the portable wireless device moves from the one base station to the next. So in order to provide with a perfect and the continuous communication by the proper selection of the network the paper put forward the PSO in the selection handling the hand off initiation along with the GRA based network selection to provide with the perfect hand off and continuous connection that leads to an enhancement in the conveyance of the information in terms of 
Journal of ISMAC (2019)

Vol.01/ No. 01

Pages: $160-171$

http://irojournals.com/iroismac/

DOI: https://doi.org/10.36548/jismac.2019.3.003

the throughput and the delay. The fig. 4 below shows the initiation of the handover and the decision making in the network selection to have a perfect network selection and a vertical handover.

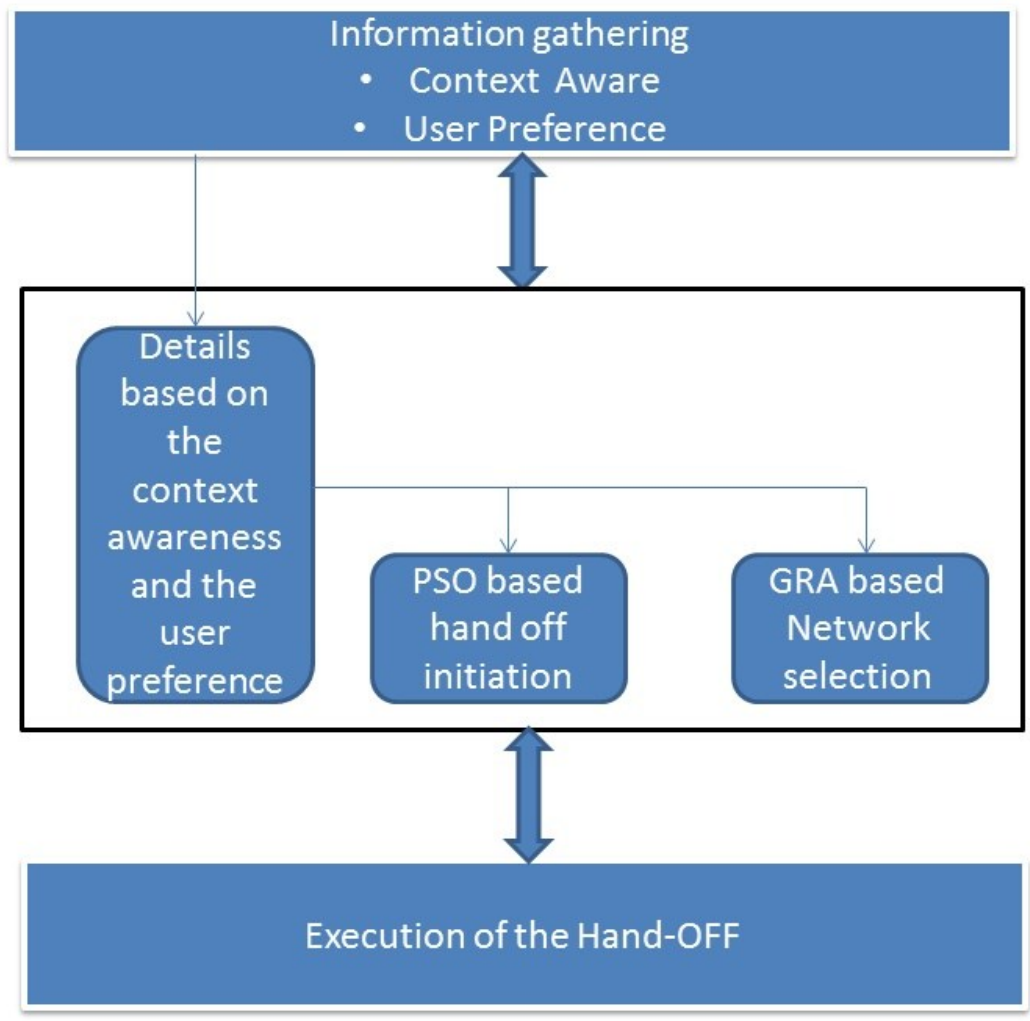

Fig.4 Hand-OFF initiation and Network Selection

The fig. 4 shows the proposed hand-off initiation and the network selection using the PSO and the network selection using the GRA. Before proceeding with the initiation and the network selection the information's related to the initiation of the handover that is context aware and based on the user preference is gathered. The information's about the networks available are gathered, the information's such as the link quality $\left(L_{q}\right)$, network coverage $\left(\mathrm{Co}_{n}\right)$, cost $\left(C_{n}\right)$ and the quality of the service $\left(Q O S_{n}\right)$ rendered by the network are gathered, the life span of the network $\left(L_{s p}\right)$, further the status of the network $\left(S_{n}\right)$, its velocity $\left(V_{n}\right)$ are all gathered along with the user preferences based on the priorities $(\mathrm{Pr})$ and the primary objectives $(\mathrm{Prm})$. The information gathered are utilized in enumerating the network with the maximum performance enhancement utilizing the PSO [16] and further the network is ranked using the 
Journal of ISMAC (2019)

Vol.01/ No. 01

Pages: $160-171$

http://irojournals.com/iroismac/

DOI: https://doi.org/10.36548/jismac.2019.3.003

GRA [7] and utilized for handover. The algorithm in the fig.5 below shows the steps involved in the decision making and the selection of the network for the vertical hand over.

Input: network information's

Output: Hand-OFF decision

For all the networks available

Start

Gather the network information's $L_{q}, C_{n}, \mathrm{Co}_{n}, Q O S_{n}, S_{n}, V_{n}, L_{s p}$, Pr, Prm

Enumerate the network performance

Apply the particle swarm optimization

Determine network with $\operatorname{MAX}\left(L_{q}, \operatorname{Co}_{n}, Q O S_{n}, S_{n}, V_{n}, L_{s p}\right)$ and $\operatorname{Min}\left(C_{n}\right)$

Sort the network in ascending order starting from the optimal one

Apply grey relational analysis

Determine the score using the $S C_{x}=\prod_{i=1}^{N} y_{x i}^{w i} / /$ where $\mathrm{x}$ is the attributes and the $\mathrm{w}$ is the weight

Calculate grey relational co-efficient// describes similarity between each candidate in the network and the ideal network.

Select the network based on the $N_{s}=\arg _{\max } x_{x \in C_{a}} G R C_{o, x} / /$ where $C_{a}$ is the number of the candidate network

Rank the network

Stop

Execute Hand-OFF

Fig.5 Proposed Algorithm for Vertical Hand-OFF decision

The algorithm utilizing the PSO in the hand-off initiation enumerates the network performance based on the information gathered and sorts out the network in the ascending order. Further the grey relational analysis once again calculates the rank of the network based on the sorting provided by the PSO to selects the perfect network for the handover to proceed with the execution of the handover. The proposed method provides a continuous and a perfect communication at a high speed utilizing the scheduling strategy for the conveyance and the switching of the services through the network that is designated.

\section{PERFORMANCE ANALYSIS}


Journal of ISMAC (2019)

Vol.01/ No. 01

Pages: $160-171$

http://irojournals.com/iroismac/

DOI: https://doi.org/10.36548/jismac.2019.3.003

The performance analysis of the proposed method utilizing the PSO and the GRA is done using the network simulator-2 to understand the performance enhancement achieved in the heterogeneous wireless networks. The evaluation of the three methods (Proposed, TOPSIS and SAW) is done to note down the improvements attained in the network using the proposed method for various average connection times in terms of the through put, latency and cost. This vertical hand-off decision being an interactive one as it based on the user requirement and the context awareness the proposed method of PSO-GRA shows a better performance than the TOPSIS and the SAW in terms of the delay, and the throughput

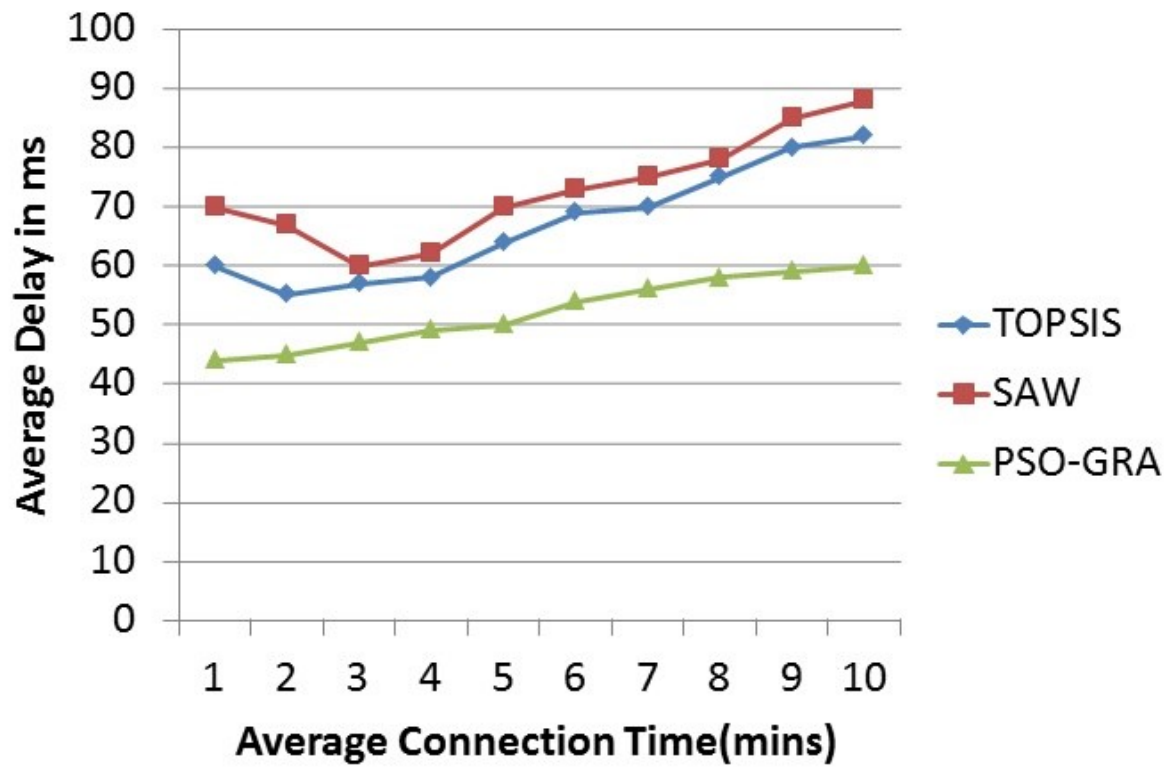

The fig. 6 above shows the delay incurred in the information transmission based on the proposed system and the other two methods TOPSIS and the SAW. The results shows that the proposed method shows a reduce delay for the interactive traffic classes compared to the SAW and the TOPSIS. 
Journal of ISMAC (2019)

Vol.01/ No. 01

Pages: $160-171$

http://irojournals.com/iroismac/

DOI: https://doi.org/10.36548/jismac.2019.3.003

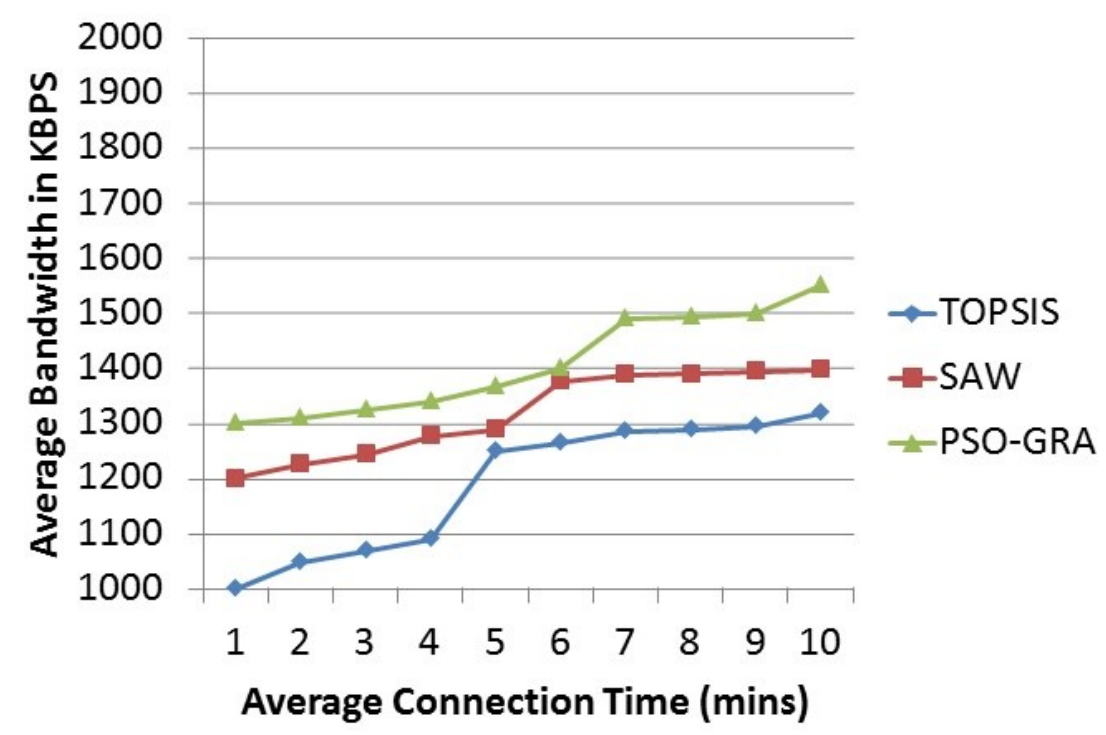

Fig .7 Average Bandwidth

The fig.7 above shows the average bandwidth of the network. The GRA provides with a higher bandwidth compared to the other two methods for the traffic class that is interactive and the results proves that the proposed method is provides with a higher bandwidth paving way for a maximized throughput compared to the SAW and the TOPSIS. 
Journal of ISMAC (2019)

Vol.01/ No. 01

Pages: $160-171$

http://irojournals.com/iroismac/

DOI: https://doi.org/10.36548/jismac.2019.3.003

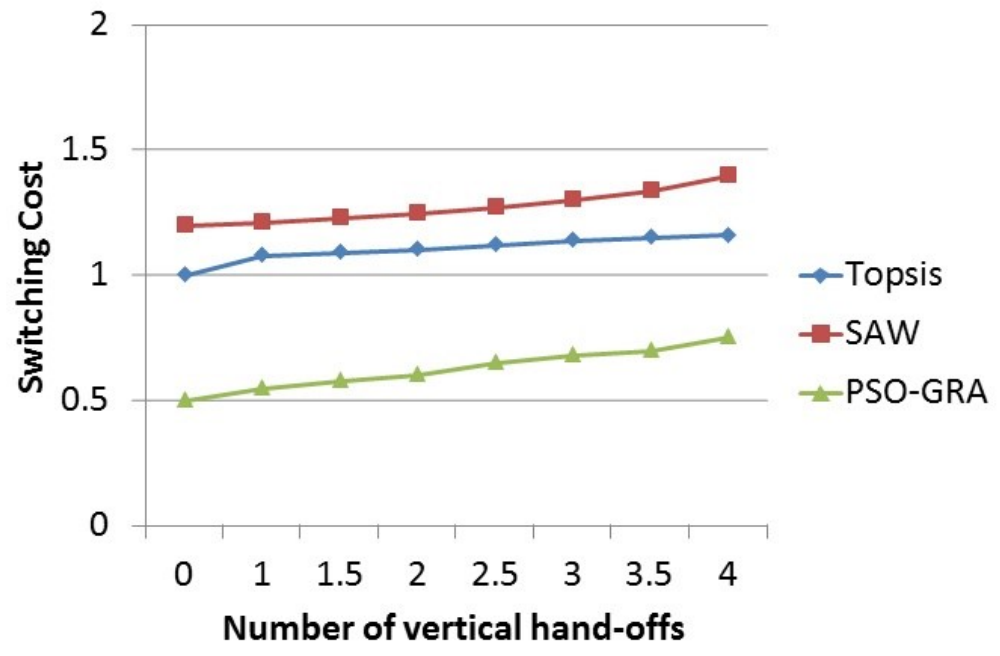

Fig .8 Switching Cost

The fig. 8 above provides the details of the cost of the switching for the number of vertical hand overs based on the grey-relational analysis integrated particle swarm optimization, and the technique for order preference by similarity to ideal solution, and the simple additive weighting.

\section{CONCLUSION}

The paper provides an enhanced vertical hand-off along with the perfect network selection schema for the heterogeneous wireless network utilizing the PSO as an initiation of the hand-off enumerating the optimal one based on its attributes and the ranking of the network using the grey relational analysis, calculating the rank for the each network based on the score. The network selected is used with the execution of the hand over providing using the scheduling strategies for the conveyance and the switching of the services through the network that is designated. The performance analysis of the proposed for the heterogeneous wireless network handover provides an enhanced service in terms of the delay, throughput and the cost. 
Journal of ISMAC (2019)

Vol.01/ No. 01

Pages: $160-171$

http://irojournals.com/iroismac/

DOI: https://doi.org/10.36548/jismac.2019.3.003

\section{References}

[1] Han, Zhu, and KJ Ray Liu. Resource allocation for wireless networks: basics, techniques, and applications. Cambridge university press, 2008.

[2] Fitzek, Frank HP, and Marcos D. Katz. Cooperation in wireless networks: principles and applications. New York: Springer, 2006.

[3] Khan, Athar Ali, Mubashir Husain Rehmani, and Yasir Saleem. "Neighbor discovery in traditional wireless networks and cognitive radio networks: Basics, taxonomy, challenges and future research directions." Journal of Network and Computer Applications 52 (2015): 173-190.

[4] Wang, Lusheng, and Geng-Sheng GS Kuo. "Mathematical modeling for network selection in heterogeneous wireless networks-A tutorial." IEEE Communications Surveys \& Tutorials 15, no. 1 (2012): 271-292.

[5] $\mathrm{Xu}, \mathrm{Ke}, \mathrm{Yi} \mathrm{Qu}$, and Kun Yang. "A tutorial on the internet of things: from a heterogeneous network integration perspective." IEEE Network 30, no. 2 (2016): 102-108.

[6] Kassar, Meriem, Brigitte Kervella, and Guy Pujolle. "An overview of vertical handover decision strategies in heterogeneous wireless networks." computer communications 31, no. 10 (2008): 2607-2620.

[7] Stevens-Navarro, Enrique, and Vincent WS Wong. "Comparison between vertical handoff decision algorithms for heterogeneous wireless networks." In 2006 IEEE 63rd Vehicular Technology Conference, vol. 2, pp. 947-951. IEEE, 2006.

[8] Niyato, Dusit, and Ekram Hossain. "Dynamics of network selection in heterogeneous wireless networks: An evolutionary game approach." IEEE transactions on vehicular technology 58, no. 4 (2008).

[9] Yan, Xiaohuan, Y. Ahmet Şekercioğlu, and Sathya Narayanan. "A survey of vertical handover decision algorithms in Fourth Generation heterogeneous wireless networks." Computer networks 54, no. 11 (2010): 1848-1863.

[10] Stevens-Navarro, Enrique, Yuxia Lin, and Vincent WS Wong. "An MDP-based vertical handoff decision algorithm for heterogeneous wireless networks." IEEE Transactions on Vehicular Technology 57, no. 2 (2008): 1243-1254.

[11]Zahran, Ahmed H., Ben Liang, and Aladdin Saleh. "Signal threshold adaptation for vertical handoff in heterogeneous wireless networks." Mobile Networks and Applications 11, no. 4 (2006): 625-640.

[12] Bari, Farooq, and Victor CM Leung. "Automated network selection in a heterogeneous wireless network environment." IEEE network 21, no. 1 (2007): 34-40.

[13] Ormond, Olga, Philip Perry, and John Murphy. "Network selection decision in wireless heterogeneous networks." In 2005 IEEE 16th International Symposium on Personal, Indoor and Mobile Radio Communications, vol. 4, pp. 2680-2684. IEEE, 2005. 
Journal of ISMAC (2019)

Vol.01/ No. 01

Pages: $160-171$

http://irojournals.com/iroismac/

DOI: https://doi.org/10.36548/jismac.2019.3.003

[14] Sgora, Aggeliki, Dimitrios D. Vergados, and Periklis Chatzimisios. "An access network selection algorithm for heterogeneous wireless environments." In The IEEE symposium on Computers and Communications, pp. 890-892. IEEE, 2010.

[15]Lei, Lei, Zhangdui Zhong, Kan Zheng, Jiadi Chen, and Hanlin Meng. "Challenges on wireless heterogeneous networks for mobile cloud computing." IEEE Wireless Communications 20, no. 3 (2013): $34-44$.

[16] Kennedy, James. "Particle swarm optimization." Encyclopedia of machine learning (2010): 760-766. 